\title{
Agiles, efficients et en évolution : la réponse rapide des Centres de collaboration nationale à la COVID-19 au Canada
}

\author{
Maureen Dobbins, Ph. D. (1); Alejandra Dubois, Ph. D. (2); Donna Atkinson, M.A. (3); Olivier Bellefleur, M. Sc. (4); \\ Claire Betker, Ph. D. (5); Margaret Haworth-Brockman, M. Sc. (6); Lydia Ma, Ph. D. (7)
}

Publié en ligne le 17 février 2021

Diffuser cet article sur Twitter

\section{Résumé}

Depuis décembre 2019, on assiste à une explosion mondiale de la recherche sur la COVID-19. Au Canada, les six Centres de collaboration nationale (CCN) en santé publique constituent l'un des piliers de la prise de décisions informées par les données probantes, car ils recueillent, résument et traduisent les connaissances émergentes. Financés par l'Agence de la santé publique du Canada et répartis sur le territoire, ils favorisent et soutiennent l'utilisation des résultats de la recherche scientifique et d'autres connaissances pour renforcer les pratiques, les programmes et les politiques en santé publique. Cet article fournit un aperçu de la manière dont les CCN participent à la mobilisation des connaissances en santé publique au Canada, met en évidence leur contribution à la lutte contre la COVID-19 et décrit les nombreuses difficultés rencontrées.

Mots-clés : mobilisation des connaissances, COVID-19, SRAS-CoV-2, réseaux de connaissances, pratique de santé publique, pratique fondée sur des données probantes, prise de décisions organisationnelles, maladies infectieuses émergentes

\section{Introduction}

L'émergence du SRAS-CoV-2 vers la fin de 2019 a entraîné une pandémie qui a mené, entre autres choses, à une explosion sans précédent de la recherche et à un déluge d'information dans le journalisme scientifique populaire et la presse grand public. L'évolution constante des connaissances et de l'information liées au virus a considérablement nui à la capacité des responsables politiques et des autres décideurs à utiliser les meilleures données probantes disponibles. En outre, les activités de collecte, de synthèse et de traduction des nouvelles données probantes fondées sur la science concernant la COVID-19 se sont avérées particulièrement complexes. La croissance exponentielle des données et de l'information a rendu de plus en plus ardu de trouver rapidement des données probantes suffisamment fiables pour éclairer les décisions relatives aux politiques et aux pratiques. Aux prises avec ces enjeux, des occasions se sont présentées pour mettre à profit une approche de mobilisation des connaissances concertée, en tenant compte des connaissances, des compétences, de l'expertise, des capacités et des réseaux de chacun des six Centres de collaboration nationale $(\mathrm{CCN})$ en santé publique au Canada.

Si de nombreuses organisations ont largement contribué à la réponse en santé publique à la COVID-19, cet article portera spécifiquement sur les six CCN. Fondés en $2005^{1}$ à la suite de la première épidémie de syndrome respiratoire aigu sévère (SRAS) au Canada, les CCN ont pour mission principale de mobiliser rapidement et
Points saillants

- L'explosion de la recherche menée sur la COVID-19 au Canada et ailleurs dans le monde a nécessité une augmentation de la capacité à soutenir la prise de décisions informées par les données probantes.

- Parmi les divers mécanismes préconisés par le Canada pour atteindre cet objectif, les Centres de collaboration nationale $(\mathrm{CCN})$ en santé publique jouent un rôle essentiel pendant la pandémie pour soutenir la prise de décisions informées par les données probantes.

- Les CCN, qui constituent un réseau de réseaux s’appuyant sur six centres de connaissances, ont des liens étroits avec plusieurs partenaires provinciaux, territoriaux, locaux et internationaux.

- Pour lutter contre la COVID-19, les CCN renforcent de façon significative les connaissances, les compétences et les capacités en santé publique et soutiennent les professionnels en santé publique en synthétisant des connaissances fondées sur des données probantes pour leur intégration aux politiques et aux pratiques.

efficacement des connaissances rigoureuses à l'intention des décideurs en santé publique au Canada en cas de crise nationale ou mondiale ${ }^{2}$.

\section{Rattachement des auteurs :}

1. Centre de collaboration nationale des méthodes et outils, Hamilton (Ontario), Canada

2. Agence de la santé publique du Canada, Ottawa (Ontario), Canada

3. Centre de collaboration nationale de la santé autochtone, Prince George (Colombie-Britannique), Canada

4. Centres de collaboration nationale sur les politiques publiques et la santé, Montréal (Québec), Canada

5. Centre de collaboration nationale des déterminants de la santé, Antigonish (Nouvelle-Écosse), Canada

6. Centre de collaboration nationale des maladies infectieuses, Winnipeg (Manitoba), Canada

7. Centre de collaboration nationale en santé environnementale, Vancouver (Colombie-Britannique), Canada

Correspondance : Alejandra Dubois, Agence de la santé publique du Canada, 130, chemin Colonnade (indice de l'adresse 6501H), Ottawa (Ontario) K1A 0K9; tél. : 343-549-1247; courriel : alejandra.dubois@canada.ca 
Cet article vise à résumer les actions des $\mathrm{CCN}$ en soutien à la réponse en santé publique à la COVID-19 au Canada, à examiner les difficultés liées à la mobilisation rapide des connaissances et à passer en revue les leçons apprises tout au long de cette expérience. Il vise également à décrire la façon dont les réseaux étendus des $\mathrm{CCN}$ et leur capacité à nouer de nouveaux partenariats pendant la pandémie ont permis de soutenir les professionnels en santé publique partout au Canada, ce qu'une organisation à elle seule n'aurait pu accomplir.

\section{Les Centres de collaboration nationale en santé publique}

Les CCN, financés par l'Agence de la santé publique du Canada, sont répartis à travers le Canada (figure 1). Ils ont pour mission de favoriser et de soutenir l'utilisation des données probantes issues de la recherche scientifique et d'autres connaissances pour renforcer les pratiques, les programmes et les politiques en santé publique au Canada, et ce, dans certains domaines précis en santé publique : déterminants de la santé, santé environnementale, politiques publiques et santé, santé autochtone, maladies infectieuses et méthodes et outils (tableau 1).

Les CCN s'acquittent de leur mission en favorisant la collaboration et le réseautage entre différents intervenants et en mettant à profit les expertises régionales, nationales et internationales pour renforcer les connaissances, les compétences et les capacités sur le plan individuel, organisationnel et systémique. Ils transforment les résultats de recherche et divers autres renseignements et données probantes en produits

FIGURE 1

Emplacement des Centres de collaboration nationale en santé publique au Canada

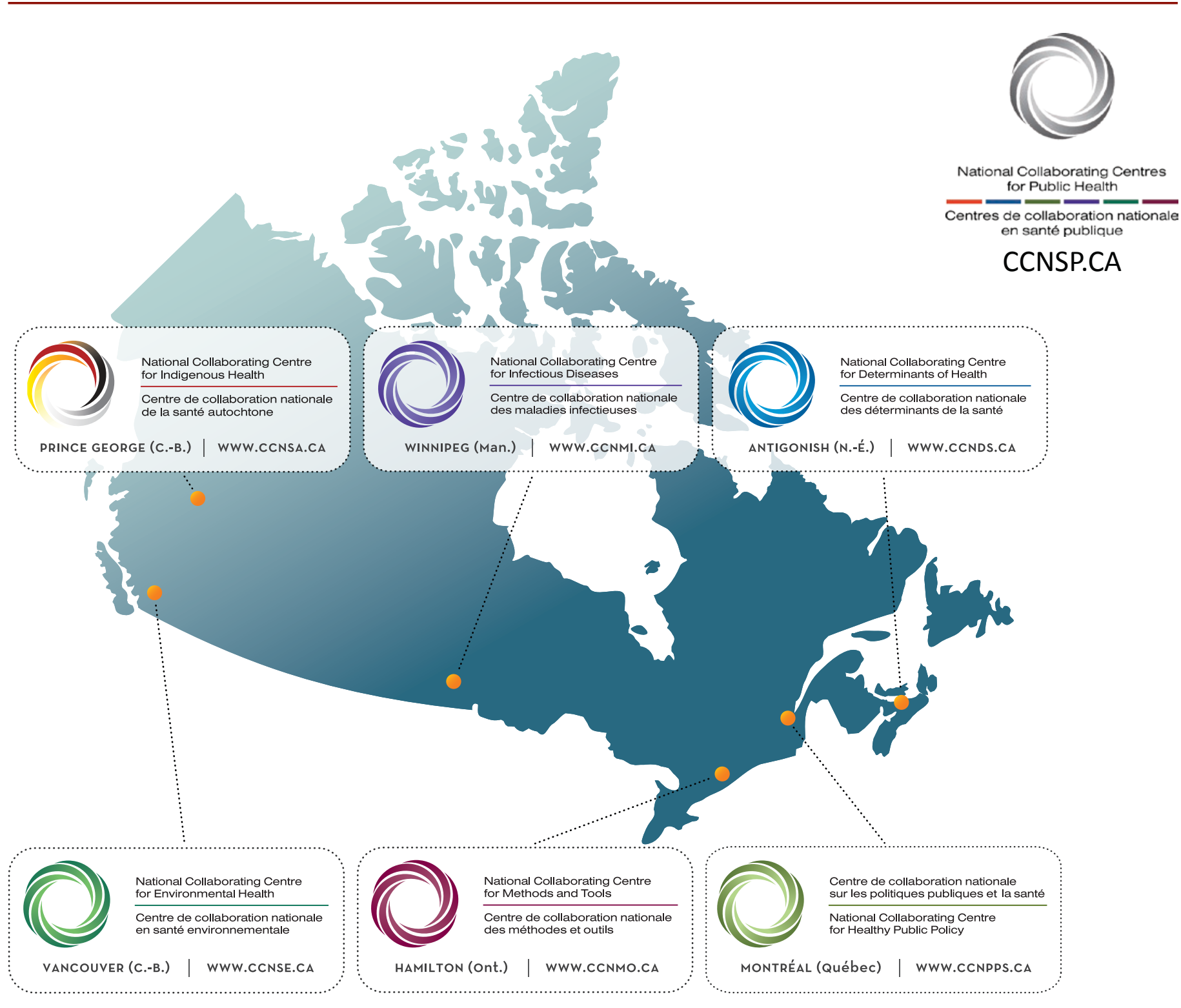

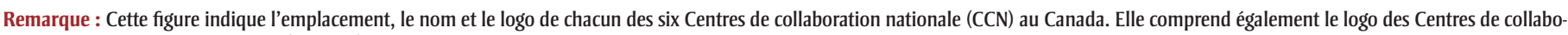
ration nationale en santé publique (ccnsp.ca), qui englobe les six CCN. 
TABLEAU 1

Centres de collaboration nationale en santé publique du Canada

\begin{tabular}{|c|c|c|c|c|}
\hline Nom du CCN & Sigle & $\begin{array}{c}\text { Organisme } \\
\text { d'accueil }\end{array}$ & Lieu & Principaux domaines et priorités \\
\hline $\begin{array}{l}\text { Déterminants } \\
\text { de la santé }\end{array}$ & CCNDS & $\begin{array}{l}\text { St. Francis Xavier } \\
\text { University }\end{array}$ & $\begin{array}{l}\text { Antigonish } \\
\text { (Nouvelle- } \\
\text { Écosse) }\end{array}$ & $\begin{array}{l}\text { - Aider la santé publique à s'attaquer aux facteurs structurels des inégalités en matière de } \\
\text { santé. } \\
\text { - Promouvoir les mesures de santé publique sur les « conditions de la vie quotidienne » qui } \\
\text { influencent la santé et l'équité, informées par les données probantes. } \\
\text { - Soutenir une « culture de l'équité » dans les organisations de santé publique et le système } \\
\text { de santé. } \\
\text { - Contribuer aux nouvelles méthodes et aux outils d'application des connaissances pour } \\
\text { faire progresser l'équité. }\end{array}$ \\
\hline $\begin{array}{l}\text { Santé } \\
\text { environnementale }\end{array}$ & CCNSE & $\begin{array}{l}\text { British Columbia } \\
\text { Centre for } \\
\text { Disease Control }\end{array}$ & $\begin{array}{l}\text { Vancouver } \\
\text { (Colombie- } \\
\text { Britannique) }\end{array}$ & $\begin{array}{l}\text { - Mieux faire connaître et mieux comprendre (1) les menaces et les avantages envi- } \\
\text { ronnementaux existants et émergents et (2) la façon d'atténuer ces menaces et d'optimiser } \\
\text { ces avantages. } \\
\text { - Traduire et mettre en évidence les résultats de recherche qui éclairent une pratique efficace } \\
\text { en santé environnementale. } \\
\text { - Mettre en commun l'expérience des praticiens de la santé environnementale de l'ensemble } \\
\text { du Canada pour éclairer une pratique qui est efficace et adaptée à l'orientation évolutive } \\
\text { de la santé publique. }\end{array}$ \\
\hline $\begin{array}{l}\text { Maladies } \\
\text { infectieuses }\end{array}$ & CCNMI & $\begin{array}{l}\text { University of } \\
\text { Manitoba }\end{array}$ & $\begin{array}{l}\text { Winnipeg } \\
\text { (Manitoba) }\end{array}$ & $\begin{array}{l}\text { - Maladies émergentes et éclosions. } \\
\text { - Tuberculose. } \\
\text { - Modélisation mathématique pour la santé publique. } \\
\text { - Prévention et contrôle du VIH et des infections transmissibles sexuellement et par le sang. } \\
\text { - Utilisation des antimicrobiens et résistance aux antimicrobiens. } \\
\text { - Migration et mobilité de la population. } \\
\text { - Points saillants (qui proposent aux lecteurs des orientations cliniques et en santé publique, } \\
\text { des données probantes et d'autres sources d'information). } \\
\text { - Répertoire des maladies à déclaration obligatoire. }\end{array}$ \\
\hline Santé autochtone & CCNSA & $\begin{array}{l}\text { University of } \\
\text { Northern British } \\
\text { Columbia }\end{array}$ & $\begin{array}{l}\text { Prince George } \\
\text { (Colombie- } \\
\text { Britannique) }\end{array}$ & $\begin{array}{l}\text { - Accroître la compréhension et l'application des données probantes provenant des } \\
\text { Autochtones sur la santé des Premières Nations, des Inuits et des Métis tout au long de } \\
\text { leur vie afin d'appuyer la prise de décisions relatives aux politiques, aux pratiques et aux } \\
\text { programmes de santé publique. } \\
\text { - Favoriser les partenariats, les collaborations et les réseaux pour recueillir des données } \\
\text { probantes provenant des Autochtones dans l'ensemble des secteurs et des administrations } \\
\text { à l'appui de l'équité en matière de santé autochtone. }\end{array}$ \\
\hline $\begin{array}{l}\text { Politiques } \\
\text { publiques } \\
\text { et santé }\end{array}$ & CCNPPS & $\begin{array}{l}\text { Institut national } \\
\text { de santé publique } \\
\text { du Québec }\end{array}$ & $\begin{array}{l}\text { Montréal } \\
\text { (Québec) }\end{array}$ & $\begin{array}{l}\text { - Soutenir le développement des compétences et des capacités organisationnelles en analyse } \\
\text { des politiques. } \\
\text { - Soutenir la mise en œuvre d'approches intersectorielles pour promouvoir des politiques } \\
\text { publiques favorables à la santé. } \\
\text { - Élaborer des approches politiques pour aborder les enjeux émerónts en santé publique. }\end{array}$ \\
\hline
\end{tabular}

Abréviation : CCN, Centre de collaboration nationale.

de connaissance adaptés à divers publics, mis en contexte et disponibles dans les deux langues officielles. Ils travaillent avec un grand nombre de partenaires et d'organisations à l'échelle du pays pour créer des occasions de partage de connaissances et d'apprentissage mutuel ${ }^{3}$.

\section{La contribution des $\mathrm{CCN}$ à la réponse du Canada à la COVID-19}

Même si chaque CCN est unique et a ses propres centres d'intérêt, caractéristiques et expertises, leur agilité et leur capacité à répondre aux enjeux émergents constituent un dénominateur commun. Celui-ci les positionne particulièrement bien pour soutenir le système de la santé publique dans ses efforts d'intervention. En effet, dès le début de la pandémie de COVID-19, chaque $\mathrm{CCN}$ a réorienté ses priorités pour 
répondre aux besoins des professionnels en santé publique en matière de données probantes et pour combler les lacunes lorsqu'elles se présentaient.

Parmi les ressources clés produites par les $\mathrm{CCN}$ figurent des listes ou des répertoires en ligne de données probantes sur la COVID-19 portant sur des sujets spécifiques à l'intérieur des domaines d'expertise de chaque $\mathrm{CCN}^{4-8}$; des synthèses de données probantes sur des questions prioritaires cernées par les décideurs et les praticiens en santé publique ${ }^{9-13}$; un document d'information sur le SRAS-CoV-2 qui présente des notions élémentaires sur sa virologie et sa transmission dans le but d'éclairer la prise de mesures visant à atténuer la propagation du virus ${ }^{14}$; de nouvelles pages Internet qui répertorient des sites portant sur des sujets précis et comportant des renseignements fiables sur la COVID-1915; des ressources de modélisation mathématique ${ }^{16}$ et des fiches d'information ${ }^{17}$. Les principales activités menées par les CCN en faveur de la mobilisation des connaissances fondées sur des données probantes et des ressources liées à la COVID-19 sont des webinaires ${ }^{18,19}$, des billets de blogue ${ }^{20}$, des documents d'orientation $^{21}$, des balados ${ }^{22,23}$ et les médias sociaux.

Les $\mathrm{CCN}$ ont contribué à plusieurs projets de recherche dans le cadre de concours de financement spécial en lien avec la COVID-19 et ils ont créé un partenariat avec les Instituts de recherche en santé du Canada pour soutenir les efforts des équipes de recherche en vue de disséminer et de favoriser l'utilisation des résultats des études sur la COVID-19 financées en 2020. Ils ont également favorisé l'établissement de divers réseaux (p. ex. le Réseau sur la COVID-19 et l'équité en santé ${ }^{24}$ et le Global Network for Health in All Policies), soutenu des communautés de pratique ${ }^{25}$ et travaillé à faciliter l'intégration d'indicateurs de bien-être dans les budgets gouvernementaux et les décisions politiques se rapportant à la phase de rétablissement de la pandémie ${ }^{26}$.

Bien que chaque $\mathrm{CCN}$ fonctionne comme une entité autonome et indépendante, les $\mathrm{CCN}$ se sont réunis régulièrement, tout au long de la pandémie, pour examiner les possibilités de collaborer à bon nombre des initiatives décrites ci-dessus, éviter le chevauchement des efforts et mettre leurs ressources en commun afin de répondre aux besoins locaux, régionaux, provinciaux, territoriaux et nationaux en matière de santé publique. Plusieurs ressources ont été élaborées de concert par deux CCN ou plus, et de nombreuses initiatives visant à disséminer des données probantes sur la COVID-19 ont été menées en partenariat par divers $\mathrm{CCN}$.

\section{Difficultés rencontrées}

Comme le coronavirus à l'origine de la pandémie mondiale était un nouvel agent pathogène, ses caractéristiques et les mécanismes de transmission de la maladie n'ont commencé à être compris qu'au début de 2020, et cette compréhension a continué à évoluer au fil du temps. Durant les six premiers mois de la pandémie, en particulier, de nouvelles données probantes ont vu le jour presque quotidiennement, rendant les synthèses désuètes avant même leur diffusion. Les prépublications, soit les articles soumis à des revues qui sont publiés avant l'évaluation par les pairs, sont devenues monnaie courante, et bon nombre d'entre elles ont été publiées malgré le peu - voire l'absence - de détails sur les méthodes de recherche utilisées, ce qui soulève des interrogations sur la fiabilité des résultats. Puisque l'agent pathogène était nouveau, les besoins en matière de données probantes de la part des responsables politiques et des autres décideurs ainsi que le délai imparti pour combler ces besoins dépassaient largement la capacité de ceux qui essayaient d'y répondre.

Il s'est également avéré difficile de déterminer quelles questions traiter d'abord et à quels besoins répondre en priorité. Par exemple, il y avait très peu de données évaluées par les pairs à synthétiser concernant la COVID-19 et la santé autochtone, en particulier d'études rédigées par des chercheurs autochtones. De plus, il a été difficile de s'assurer que les points de vue et les expériences des communautés des Premières Nations, des Inuits et des Métis soient pris en compte dans l'élaboration des politiques et la prise de décisions. L’ampleur des iniquités vécues par les peuples autochtones les a exposés à un risque accru et a nécessité une mise en contexte de l'information pour que les vulnérabilités et les déterminants spécifiques de la santé des Premières Nations, des Inuits et des Métis puissent être compris et que des interventions appropriées puissent être menées.
Parallèlement, il fallait accorder une attention proportionnelle aux besoins du personnel en santé publique et aux besoins des populations plus défavorisées (étant donné la disponibilité locale des ressources de soins de santé ou la stigmatisation et la discrimination constantes) afin d'éviter de perpétuer d'autres iniquités et inégalités en santé. En outre, l'arrivée de la COVID-19 n'a pas atténué la nécessité de fournir en temps opportun des données probantes et des connaissances sur d'autres maladies infectieuses omniprésentes (p. ex. infections transmissibles sexuellement et par le sang, tuberculose et résistance aux antimicrobiens $^{28,29}$ ) - à l'exception apparente de la grippe saisonnière ${ }^{27}$-, ainsi que sur d'autres programmes et services en santé publique.

De plus, même s'il était urgent de distribuer rapidement les produits de connaissances aux responsables politiques et aux autres décideurs, ceux-ci se sont vite retrouvés submergés par une trop grande quantité d'information ainsi que par de fausses informations. Le terme «infodémie» est réapparu pour définir ce phénomène particulier ${ }^{30}$. Sur le coup, il n'était pas évident de savoir comment ni à qui disséminer ces produits de connaissances. On a aussi constaté un important dédoublement des synthèses de données probantes (à l'échelle internationale, nationale, provinciale, régionale et locale), ainsi qu'un chevauchement des ressources élaborées en français et en anglais. Il était impossible de se tenir constamment à jour de ce que tout le monde faisait et produisait.

Nombreux sont ceux qui ont qualifié de «sprint» le rythme auquel fonctionnaient les organisations durant les six premiers mois de la pandémie. Il est devenu impossible de maintenir ce rythme : le personnel se fatiguait et montrait des signes d'épuisement professionnel, sans parler de l'effort supplémentaire qu'il a fallu consentir pour savoir comment fonctionner virtuellement. Il y a eu beaucoup à apprendre sur le travail d'équipe efficace et efficient à domicile, notamment en s'assurant que le personnel avait l'équipement nécessaire pour travailler virtuellement.

\section{Leçons apprises et stratégies émergentes}

Au cours des premiers mois de la pandémie (mars et avril 2020), le temps consacré à examiner les résultats de recherche, les recommandations et les leçons apprises 
des épidémies de SRAS et de grippe H1N1 a été bénéfique pour tirer parti des réussites et des échecs des stratégies passées. De la même façon, le bilan des premiers mois de la pandémie actuelle permet de tirer plusieurs leçons que les CCN utiliseront pour orienter les efforts visant à soutenir la réponse en cours et la réponse à moyen et à long terme contre la COVID19 ainsi que la phase de rétablissement.

Tout d'abord, il faut une réflexion prospective afin d'anticiper les prochaines étapes de la réponse à la pandémie et les besoins à venir des responsables politiques et des autres décideurs en matière de connaissances. En adoptant une approche proactive plutôt que réactive, il est plus facile de produire des synthèses de données probantes et des produits de connaissance, ainsi que de favoriser la mobilisation et la collaboration au moment des prises de décisions et d'établissement des politiques. Il est important de créer des ressources qui non seulement répondent aux besoins actuels liés à la COVID-19 mais seront également utiles après la pandémie.

Ensuite, les acteurs de la santé publique ont été fortement sollicités pour contenir la propagation du virus et atténuer ses effets immédiats sur tous les secteurs de la société depuis le début de la pandémie, mais ils ont aussi été sollicités pour contribuer aux politiques, aux programmes et aux pratiques visant à soutenir le rétablissement vers une société plus équitable, résiliente, durable et en santé. Or les efforts pour contenir le virus et composer avec ses effets immédiats n'ont pas laissé beaucoup de temps ni d'énergie aux organisations de santé publique pour jouer ce second rôle, qui est davantage axé sur la réponse à moyen et à long terme. En tant que réseau de réseaux, certains $\mathrm{CCN}$ étaient bien placés pour contribuer à la capacité de pointe nécessaire pour appuyer les acteurs de la santé publique dans leur réponse immédiate, tandis que d'autres ont pu se mobiliser pour les soutenir dans leur contribution à la réponse à moyen et à long terme.

Enfin, les relations et les partenariats établis sont essentiels. La capacité à tirer parti de la santé publique a été cruciale dans le travail des CCN, tout comme l'aptitude à puiser dans les connaissances et les expériences autochtones relativement aux pandémies passées (p. ex. H1N1, variole). Les communications régulières entre les CCN et avec l'Agence de la santé publique du Canada ont joué un rôle déterminant pour coordonner le travail, favoriser la collaboration et éviter le chevauchement des efforts. Il est nécessaire de disposer d'un personnel dévoué, ayant des relations de confiance déjà établies et pouvant travailler entre les juridictions, pour savoir qui travaille sur quoi, pour compiler les données et pour les partager.

\section{Tirer parti des réseaux et nouer de nouveaux partenariats}

Une réponse globale à la COVID-19 exige une mobilisation, une collaboration et des partenariats entre disciplines, secteurs et juridictions. S'appuyant sur des relations bien établies avec de nombreux partenaires et collègues (professionnels de la santé publique, ministères, organisations synthétisant les connaissances, chercheurs et établissements d'enseignement postsecondaire, etc.), les $\mathrm{CCN}$ ont contribué à assurer une liaison entre chercheurs, décideurs et praticiens afin de faciliter le partage des connaissances ainsi que l'avènement de politiques, de décisions, de pratiques et de nouvelles recherches fondées sur des données probantes.

Que ce soit en tant que participants ou en tant que responsables, les CCN ont pris une part active aux initiatives de collaboration nationale et internationale issues de la pandémie. L'une de ces initiatives est le réseau COVID-19 Evidence Network to support Decision-making (COVID-END) ${ }^{31}$. Ce réseau international aide les organisations qui soutiennent la prise de décisions à trouver et à utiliser les meilleures données probantes sur la COVID-19, facilite la coordination des efforts de synthèse des connaissances à l'échelle mondiale ${ }^{32}$ et réduit le chevauchement des efforts. En participant au réseau COVID-END, les CCN contribuent à l'écosystème des données probantes et évitent le dédoublement des synthèses de connaissances.

\section{Conclusion}

La santé publique informée par les données probantes est issue des ouvrages précurseurs d'Archie Cochrane qui, dès le début des années 1970, avait constaté que l'efficacité de nombreux traitements médicaux manquait de fondements scientifiques $^{33}$. Au fil des ans, le Canada et de nombreux autres pays ont développé leur capacité à synthétiser les données probantes pour améliorer l'usage des données scientifiques dans les pratiques, les politiques et les décisions courantes en matière de santé publique. Chaque pandémie (SRAS, H1N1, COVID-19) a suscité un engagement croissant à l'échelle nationale et internationale envers une réponse informée par les données probantes. En fait, c'est l'épidémie de SRAS de 2003 qui a donné lieu à la création de l'Agence de la santé publique du Canada, au Réseau pancanadien de santé publique et aux CCN comme piliers structurels du système de santé publique canadien.

Au cours des 16 années qui ont suivi leur création, les $\mathrm{CCN}$ ont fait leurs preuves et ont démontré qu'ils pouvaient travailler avec les autres piliers, soutenir la santé publique et répondre à ses besoins grâce aux données probantes, aux systèmes de connaissances et en établissant des réseaux. Aujourd'hui, nous constatons les avantages de l'investissement dans les CCN, qui remplissent un rôle essentiel dans le système en santé publique du Canada pendant cette pandémie en cernant les lacunes, en compilant et en synthétisant les données probantes et en facilitant la mobilisation et l'échange des connaissances afin de combler le fossé entre les données probantes, les politiques et les pratiques.

\section{Remerciements}

Les Centres de collaboration nationale reçoivent du financement de l'Agence de la santé publique du Canada. Nous tenons à remercier sincèrement les membres clés de chacun des Centres de collaboration nationale en santé publique d'avoir examiné les ébauches du manuscrit et de nous avoir fait part de leurs commentaires (par ordre alphabétique : Emily Clark, Margo Greenwood, Heather Husson, Michael Keeling, Yoav Keynan, Tom Kosatsky et Sarah Neil-Sztramko). Nous remercions également la $D^{\text {re }}$ Patricia Huston pour ses conseils et sa contribution à la première ébauche du manuscrit.

\section{Contribution des auteurs et avis}

$\mathrm{AD}$ était responsable de la conceptualisation, de la méthodologie et de l'administration du projet, ainsi que de la rédaction, de la révision et de la correction de la première ébauche. MD a révisé en profondeur la première ébauche et a ajouté plusieurs nouvelles sections en fonction des commentaires des évaluateurs. Tous les autres auteurs cités ont validé la conception de la 
recherche, ainsi que révisé et corrigé les deux ébauches du manuscrit. Tous les auteurs ont contribué à la révision critique de l'article tout au long du processus d'élaboration et d'évaluation par les pairs.

Le contenu de l'article et les points de vue qui y sont exprimés n'engagent que les auteurs; ils ne correspondent pas nécessairement à ceux du gouvernement du Canada.

\section{Conflits d'intérêts}

Aucun.

\section{Références}

1. Centres de collaboration nationale en santé publique [Internet]; 2020 [consultation le 21 janv. 2021]. En ligne à : https://ccnsp.ca

2. Medlar B, Mowat D, Di Ruggiero E, Frank J. Introducing the National Collaborating Centres for Public Health. CMAJ. 2006;175(5):493-494. https://doi.org/10.1503/cmaj.060850

3. Dubois A, Lévesque M. Les Centres de collaboration nationale du Canada : faciliter la prise de décisions informées par des données probantes en santé publique. Relevé des maladies transmissibles au Canada. 2020;46(2/3): 35-39. http://doi.org/10.14745/ccdr .v46i23a02f

4. Centre de collaboration nationale en santé environnementale (CCNSE). Ressources de santé environnementale pour la pandémie de COVID-19 [Internet]. Vancouver (C.-B.) : CCNSE; 2020 [modification le 16 déc. 2020; consultation le 21 janv. 2021]. En ligne à : https://ccnse.ca/environmental-health -in-canada/health-agency-projects /ressources-de-sante-environnementale -pour-la

5. Centre de collaboration nationale des méthodes et outils (CCNMO). Revues rapides de données probantes sur la COVID-19 [Internet]. Hamilton (Ont.) : CCNMO; 2020 [modification en janv. 2021; consultation le 21 janv. 2021]. En ligne à : https://www.nccmt.ca/fr /covid-19/covid-19-revues-rapides

6. Centre de collaboration nationale de la santé autochtone (CCNSA). Mises à jour sur la COVID-19 [Internet]. Prince George (C.-B.) : CCNSA; 2021 [consultation le 21 janv. 2021]. En ligne à : https://www.ccnsa.ca/fr/ListingIndex .aspx? sortcode $=1.6 .17$ \&id $=451$
7. Centre de collaboration nationale sur les politiques publiques et la santé (CCNPPS). Éthique en santé publique et COVID-19 : ressources sélectionnées [Internet]. Montréal (Qc) : CCNPPS; 2020 [consultation le 21 janv. 2021]. En ligne à : http://www.ccnpps.ca/822 /covid-19--ressources-selectionnees .ccnpps

8. Centre de collaboration nationale de la santé autochtone (CCNSA). Collection sur la COVID-19 [Internet]. Prince George (C.-B.) : CCNSA; 2020 [consultation le 21 janv. 2021]. En ligne à : https:// www.ccnsa.ca/fr/publicationsExternal .aspx? sortcode $=1.19 .0 .3 \&$ Collectionid $=1$

9. Eykelbosh A. Précautions concernant la COVID-19 dans les immeubles à logements multiples [Internet]. Vancouver (C.-B.) : Centre de collaboration nationale en santé environnementale; 2020 [consultation le 21 janv. 2021]. En ligne à : https://ccnse.ca/documents /guide/precautions-concernant-la-covid -19-dans-les-immeubles-logements -multiples

10. Chen T, O’Keeffe J. La COVID-19 dans les espaces clos - Mesures de désinfection de l'air et des surfaces [Internet]. Vancouver (C.-B.) : Centre de collaboration nationale en santé environnementale; 2020 [consultation le 21 janv. 2021]. En ligne à : https:// ccnse.ca/documents/guide/la-covid -19-dans-les-espaces-clos-mesures-de -desinfection-de-lair-et-des-surfaces

11. Freeman S, Eykelbosh A. La COVID-19 et la sécurité à l'extérieur : considérations sur l'utilisation des espaces récréatifs extérieurs [Internet]. Vancouver (C.-B.) : Centre de collaboration nationale en santé environnementale; 2020 [consultation le 21 janv. 2021]. En ligne à : https://ccnse.ca/documents /guide/la-covid-19-et-la-securite -lexterieur-considerations-sur-lutilisation -des-espaces

12. O’Keeffe J. Chant choral : risques et précautions associés à la COVID-19 [Internet]. Vancouver (C.-B.) : Centre de collaboration nationale en santé environnementale; 2020 [consultation le 21 janv. 2021]. En ligne à : https:// ccnse.ca/documents/evidence-review /chant-choral-risques-et-precautions -associes-la-covid-19
13. Centre de collaboration nationale des méthodes et outils (CCNMO). COVID19 Service rapide de données probantes - À propos [Internet]. Hamilton (Ont.) : CCNMO; 2020 [consultation le 21 janv. 2021]. En ligne à : https:// www.nccmt.ca/fr/covid-19/covid-19 -rapid-evidence-service

14. O’Keeffe J, Freeman S, Nicol A-M. La transmission du sars-CoV-2 : faits saillants [Internet]. Vancouver (C.-B.) : Centre de collaboration nationale en santé environnementale; 2020 [consultation le 20 janv. 2021]. En ligne à : https://ccnse.ca/documents/evidence -review/la-transmission-du-sars-cov -2-faits-salliants

15. Centre de collaboration nationale des maladies infectieuses (CCNMI). Points saillants : Éclosion de la maladie à coronavirus (COVID-19) [Internet]. Winnipeg (Man.) : CCNMI; 2020 [consultation le 16 janv. 2021]. En ligne à : https://ccnmi.ca/eclosion-du-nouveau -coronavirus/

16. Centre de collaboration nationale des maladies infectieuses (CCNMI). Modèles COVID-19 de l'Agence de la santé publique du Canada [Internet]. Winnipeg (Man.) : CCNMI; [date inconnue; consultation le 16 janv. 2021]. En ligne à : https://ccnmi.ca/modeles-covid-19/

17. Centre de collaboration nationale de la santé autochtone (CCNSA). Maintenir la santé et le bien-être des enfants et des adolescents des Premières Nations, des Inuits et des Métis pendant la COVID-19 [Internet]. Prince George (C.-B.) : CCNSA; 2020 [consultation le 21 janv. 2021]. En ligne à : https:// www.ccnsa.ca/fr/publicationsview .aspx? sortcode $=1.8 .21 .0 \& \mathrm{id}=296$

18. National Collaborating Centre for Infectious Diseases (NCCID). Synergies in infectious disease modelling for public health [Internet]. Winnipeg (MB): NCCID; [date inconnue; consultation le 16 janv. 2021]. En ligne à : https:// nccid.ca/synergies/

19. Centre de collaboration nationale des déterminants de la santé (CCNDS). Ateliers et activités : Série de discussion : l'équité en santé, les déterminants de la santé et la COVID-19 [Internet]. Antigonish (N.-É.) : CCNDS; 2020 [Consultation le 18 janv. 2021]. En ligne à : https://nccdh.ca/fr/workshops -events/entry/COVID-19-webinar -conversation-series 
20. Centre de collaboration nationale des déterminants de la santé (CCNDS). Blogue : COVID-19 [Internet]. Antigonish (N.-É.) : CCNDS; 2020 [consultation le 18 janv. 2021]. En ligne à : https:// nccdh.ca/fr/blog/type/category /covid-19

21. Haworth-Brockman M, Betker C. Mesurer ce qui compte en plein cœur de la pandémie de COVID-19 - Indicateurs d'équité pour la santé publique. Winnipeg (Man.) : Centre de collaboration nationale des maladies infectieuses; 2020. 35 p.

22. National Collaborating Centre for Infectious Diseases (NCCID). Infectious questions: what health professionals should know about COVID-19 (2019$\mathrm{nCoV}$ ) [Internet]. Winnipeg (MB): NCCID; 2021 [consultation le 16 janv. 2021]. En ligne à : https://nccid.ca /podcast-2019-ncov

23. Centre de collaboration nationale de la santé autochtone (CCNSA). Base de connaissance et publications - Baladodiffusion [Internet]. Prince George (C.-B.) : CCNSA; 2020 [consultation le 21 janv. 2021]. En ligne à : https:// www.ccnsa.ca/fr/publications.aspx ? sortcode $=1.8 .21$

24. Centre de collaboration nationale des déterminants de la santé (CCNDS). Réseau sur la COVID-19 et l'équité en santé [Internet]. Antigonish (N.-É.) : CCNDS; 2020 [consultation le 18 janv. 2021]. En ligne à : https://nccdh.ca /fr/connect/covid-19-and-health -equity-network/

25. National Collaborating Centre for Infectious Diseases (NCCID). En ligne à : Winnipeg (MB): NCCID; 2020 [consultation le 21 janv. 2021]. En ligne à : https://nccid.ca/mod4ph/

26. National Collaborating Centre for Healthy Public Policy (NCCHPP). Webinar-wellbeing budgeting and public health: promising practice for pandemic recovery? [Internet]. Montréal (QC) : NCCHPP; 2020 [consultation le 21 janv. 2021]. En ligne à : https:// www.ncchpp.ca/554/presentations .ccnpps?id_article $=2083$

27. Pierce A, Haworth-Brockman M, Marin D, Rueda ZV, Keynan Y. Changes in the incidence of seasonal influenza in response to COVID-19 social distancing measures: an observational study based on Canada's national influenza surveillance system. Research Square [prépublication]; 2020. https://doi.org /10.21203/rs.3.rs-93953/v1

28. Long $\mathrm{R}$, King $\mathrm{M}$, Doroshenko $\mathrm{A}$, Heffernan C. Tuberculosis and COVID19 in Canada. EClinicalMedicine 27; 2020:100584. https://doi.org/10.1016 /j.eclinm.2020.100584

29. Edmiston, L. The COVID-19 pandemic is threatening an HIV resurgence. The Georgia Straight [Internet]. 2020 Nov 16. En ligne à : https://www .straight.com/news/laurie-edmiston -covid-19-pandemic-is-threatening -an-hiv-resurgence

30. Merriam-Webster. Words We're Watching: 'Infodemic' [Internet]. Springfield (MA): Merriam-Webster Incorporated; 2021 [consultation le 16 janv. 2021]. En ligne à : https://www.merriam -webster.com/words-at-play/words -were-watching-infodemic-meaning

31. McMaster Health Forum. COVID-END: COVID-19 Evidence Network to support Decision-making [Internet]. Hamilton (ON): McMaster University; 2020 [consultation en ligne le 21 janv. 2021]. En ligne à : https://www .mcmasterforum.org/networks/covid -end

32. COVID-END Synthesizing Working Group. Resources and tools for researchers considering and conducting COVID19 evidence syntheses [Internet]. Hamilton (ON) : McMaster University; 2020 [consultation le 21 janv. 2021]. En ligne à : https://www.mcmasterforum .org/docs/default-source/covidend /covid-end_researchers.pdf?sfvrsn $=437 \mathrm{e} 56 \mathrm{~d} 5 \_4$

33. Brownson RC, Fielding JE, Green LW. Building capacity for evidence-based public health: reconciling the pulls of practice and the push of research. Annu Rev Public Health. 2018;39:2753. https://doi.org/10.1146/annurev -publhealth-040617-014746 